\title{
Terminology for Hormone-related Polypeptides Detected by Radioimmunoassay*
}

In a recent editorial [1] Conlon reviews the multiplicity of polypeptides which have been encountered in the pancreas, gastrointestinal tract and circulation, when antibodies raised against pancreatic glucagon are used for radioimmunoassays. The polypeptides vary in size and in their reactivity with antibodies raised against different immunogenic sites in glucagon. The author agrees with a previous proposal [2] that inter-tissue variations in molecular species are due to different proteolytic processing of the same precursor. He also attempts to establish terminological order for the complex array of polypeptides ("chaos").

It is apparent that two main types of antibodies are encountered when experimental animals are immunised with glucagon. One type binds a site containing residues 25 to 29 , close to the Cterminus of the hormone [2-4]. The other type binds a site in the $\mathrm{N}$-terminal-to-central region of the hormone, between amino acid residues 2 and 23 as mentioned by the reviewer [1]. In this case, however, studies with fragments, derivatives and a variant of glucagon indicate that the immunogenic site is probably much nearer to the centre (residues 10 to 18 ) than to the $\mathrm{N}$-terminus [2, 4]. The reviewer [1] accepts a proposal [5] that polypeptides which react with both antibodies be termed collectively immunoreactive glucagon (IRG) whereas those which bind only to the $\mathrm{N}$-terminalto-central antibodies be termed glucagon-like immunoreactivity (GLI). The molecular weight would be written as a superscript as in $\mathrm{IRG}^{3500}$. The proposed terminology is less precise than is the one which states the structures for which assay antibodies are specific and is unlikely to be accepted universally for several reasons. The adjective "immunoreactive" qualifying "glucagon" implies that there can be a glucagon which is immunoreactive and a glucagon which is not. Glucagon is a definite structural entity [6]. The suggested terminology is based on two antibodies which collectively bind only a limited proportion of the total glucagon structure. It could not describe fragments of glucagon or of glucagon precursor(s) which bind to the $\mathrm{C}$-terminal antibodies but not to the N-terminal-to-central antibodies. Also, fragments of precursors or of glucagon containing residues 1 to 12 of the hormone are not detected by either antibody [4] and consequently, are also excluded from the terminology.

The convention of using "hormone-like immunoreactivity" and "immunoreactive hormone" when materials bind one and two different antibodies, respectively, is unlikely to be extended to other polypeptide hormones and their related groups of peptides. Already, antibodies specific for more than two different regions

* The Editor-in-Chief is greatly indebted to Dr. Murphy, Professor Buchanan and others for their interest in this topic. It is only by free and open communication such as this that reasonable concensus views are obtained. Hopefully other readers will now add their own views and we may finally reach some uniformity in this perplexing field are available for cholecystokinin; Rehfeld [7] uses antibodies specific for regions 18-25, 25-30 and 29-33. Designation of the terms "immunoreactive cholecystokinin" and "cholecystokininlike immunoreactivity", to differentiate between peptides which react with antibodies specific for all, two or one of these immunogenic sites, would hardly receive favour.

Irrespective of how many individual antibodies of characterised specificity are available, it is unsafe to rely on immunoassay data alone as proof of the presence of an intact and underivatised hormone structure. When a single uncharacterised antibody, raised against an intact hormone, for example glucagon, is used for radioimmunoassay, it is reasonable to assume the detection of some polypeptide material with a limited structural similarity to glucagon. It is unsafe, however, to venture beyond the terms (i) "a material with immunoreactivity of glucagon", (ii) "glucagon-like immunoreactive polypeptide", (iii) "glucagon-like immunoreactant" (favoured by F. Sundby and A. J. Moody, Copenhagen, for material other than glucagon; personal communication) or (iv) "glucagon-like immunoreactivity". The latter two can be abbreviated to GLI which is commonly used though term (i) may be the only one which is pedantically and scientifically justifiable. GLI could be glucagon, a fragment of glucagon, a precursor of glucagon or an homologous polypeptide.

When the specificity of an assay antibody is known, it is essential to include that information. Where authors are unwilling to elaborate with terms such as "immunoreactivity detected with antibodies which are specific for glucagon ${ }_{25-29}$ ", the abbreviation " $\mathrm{G}_{25-29} \mathrm{LI}$ ", might be acceptable. The first letter " $\mathrm{G}$ " abbreviates the name of the hormone, the subscript " $19-29$ " gives the region of the hormone for which the antibody is specific and "LI" can mean "-like immunoreactivity" or "-like immunoreactant" as above. Material which reacts with antibodies which bind the central region of glucagon would then be depicted as $\mathrm{G}_{10-18} \mathrm{LI}$. Material which binds more than one antibody would be depicted showing the regional specificities of the antibodies consecutively from the $\mathrm{N}$-terminus. Glucagon, detected with both types of antibodies mentioned here, would be depicted $\mathrm{G}_{10-18,25-29} \mathrm{LI}$. The proposal that the approximate molecular weight be shown as a superscript $[1]$ is acceptable. Glucagon would then be depicted as $\mathrm{G}_{10-18}$, ${ }_{25-29} \mathrm{LI}^{3500}$ when detected using gel-filtration and the antibodies currently available. The circulating secretin fragment with molecular weight about 2500 and which binds antibody specific for the fragment secretin ${ }_{14-27}[8]$ could be depicted $\mathrm{S}_{14-27} \mathrm{LI}^{2500}$. Precursors of glucagon or fragments of precursors, encountered in the pancreas, gut and circulation $[1,2,4,9,10]$, would include $\mathrm{G}_{10-18} \mathrm{LI}^{12000}, \mathrm{G}_{10-18,25-29} \mathrm{LI}^{9000}, \mathrm{G}_{10-18} \mathrm{LI}^{8000}$ and $\mathrm{G}_{10-18} \mathrm{LI}^{4500}$.

It is essential to emphasise, that lack of detectable immunoreactivity is not an indication of the absence of a structural feature in a polypeptide $[1,2] . G_{10-18} L I^{4500}$ seems to contain the intact structure of glucagon but a C-terminal extension impedes interaction with the antibodies which are specific for glucagon $25-29$ $[1,4,9]$. 
The terminology proposed here may be convenient but as Conlon [1] accepts for the alternative it is not intended to be the "final classification". Conlon also "urges authors to compromise their rigidly held views regarding nomenclature in order to achieve a greater measure of linguistic uniformity from which a clearer understanding may develop". Rigid adherence to any proposed "linguistic uniformity" per se may not facilitate versatility and precision of expression. The Editor in Chief is strongly recommended to delay his request [11] that authors who write for this journal should use the terminology suggested by Conlon [1] until there is more consensus in the literature.

\section{R. F. Murphy}

K. D. Buchanan

\section{References}

1. Conlon JM (1980) The glucagon-like polypeptides - order out of chaos? Diabetologia 18: 1-4

2. Murphy RF (1979) The chemical characterisation of gut hormones. Clin Endocrinol Metab 8: 281-297

3. Assan RA, Slusher N (1972) Structure/function and structure immunoreactivity relationships of the glucagon molecule and related synthetic peptides. Diabetes 21: 843-855

4. Flanagan RWJ, Murphy RF, Buchanan KD (submitted for publication) Isolation and radioimmunoassay of glucagon and related peptides from human plasma. Diabetologia
5. Unger RH (1976) Glucagon symposium: report of the nomenclature committee. Metabolism 25 [Suppl 1]: ix

6. Bromer WW, Sinn LG, Behrens OK (1956) The amino acid sequence of glucagon. J Am Chem Soc 79: 2807-2810

7. Rehfeld JF (1978) Multiple molecular forms of cholecystokinin. In: Bloom SR (ed) Gut hormones. Churchill Livingstone, Edinburgh, p 213-218

8. Mason JC, Murphy RF, Henry RW, Buchanan KD (1979) Starvation induced changes in secretin-like immunoreactivity of human plasma. Biochim Biophys Acta 582: 322-331

9. Flanagan RWJ, Murphy RF, Buchanan KD (in press) Circulating forms of glucagon and related peptides in normal subjects and uremic patients. Biochem Soc Trans

10. Tager HS, Markese J (1978) Intestinal and pancreatic glucagon-like peptides. J Biol Chern 254: 2229-2233

11. Editor's note (1980) Diabetologia 18: 4

\author{
R. F. Murphy \\ K. D. Buchanan \\ Departments of Biochemistry and Medicine \\ The Queen's University \\ 97 Lisburn Road \\ Belfast BT9 7BL \\ Northern Ireland
}

